\title{
Remineralising Dentine Caries Using Sodium Fluoride with Silver Nanoparticles: An In Vitro Study
}

This article was published in the following Dove Press journal:

International Journal of Nanomedicine

\author{
Irene Shuping Zhao (D) ${ }^{1,2}$ \\ Iris Xiaoxue Yin (iD) ${ }^{2}$ \\ May Lei $\mathrm{Mei}^{3}$ \\ Edward Chin Man Lo $\mathbb{I D}^{2}$ \\ Jinyao Tang $\mathbb{D}^{4}$ \\ Quanli Li ${ }^{5}$ \\ Lok Yan So ${ }^{6}$ \\ Chun Hung Chu (iD) ${ }^{2}$ \\ 'School of Dentistry, Shenzhen \\ University Health Science Center, \\ Shenzhen, People's Republic of China; \\ ${ }^{2}$ Faculty of Dentistry, The University of \\ Hong Kong, Hong Kong, People's \\ Republic of China; ${ }^{3}$ Faculty of Dentistry, \\ University of Otago, Dunedin, New \\ Zealand; ${ }^{4}$ Department of Chemistry, The \\ University of Hong Kong, Hong Kong, \\ People's Republic of China; ${ }^{5}$ School of \\ Stomatology, Anhui Medical University, \\ Hefei, People's Republic of China; \\ ${ }^{6}$ Department of Applied Biology and \\ Chemical Technology, The Hong Kong \\ Polytechnic University, Hong Kong, \\ People's Republic of China
}

Correspondence: Chun Hung Chu Faculty of Dentistry, The University of Hong Kong, 34 Hospital Road,

Hong Kong, People's Republic of China

Tel +852-28590246

Email chchu@hku.hk
Objective: To investigate the remineralizing and staining effects of sodium fluoride $(\mathrm{NaF})$ solution with polyethylene glycol-coated silver nanoparticles (PEG-AgNPs) on artificial dentine caries.

Materials and Methods: Demineralized human dentine blocks were allocated to three groups. The blocks in group 1 underwent a topical application of a 12\% silver diamine fluoride (SDF, 14,150 ppm fluoride) solution. The blocks in group 2 received a topical application of a $2.5 \% \mathrm{NaF}$ (11,310 ppm fluoride) with PEG-AgNPs (400 ppm silver). The blocks in group 3 received deionized water. All blocks were subjected to $\mathrm{pH}$ cycling for 8 days. The surface morphology and cross-sectional features were investigated using scanning electron microscopy (SEM). The color parameters, crystal characteristics, lesion depth, and collagen degradation of the blocks were assessed using digital spectrophotometry, X-ray diffraction (XRD), microcomputed tomography, and spectrophotometry with a hydroxyproline assay, respectively.

Results: The SEM showed that dentine collagen was exposed in group 3 but not in groups 1 and 2. The mean lesion depths in groups 1 to 3 were $118 \pm 7 \mu \mathrm{m}, 121 \pm 14 \mu \mathrm{m}$, and $339 \pm 20$ $\mu \mathrm{m}$, respectively (groups $1,2<3 ; \mathrm{p}<0.001$ ). The data indicated that fluoridated PEG-AgNPs introduced no significant color effect on dentine, but SDF caused distinct discoloration. The XRD indicated that silver chloride was formed in group 1, and fluorapatite was detected in groups 1 and 2. The concentration of hydroxyproline liberated from collagen was significantly less in groups 1 and 2 than in group 3.

Conclusion: The use of NaF solution with PEG-AgNPs can remineralize artificial dentine caries and inhibit collagen degradation without causing significant tooth staining.

Keywords: silver, nanoparticles, fluoride, dentine, caries, remineralization

\section{Introduction}

Dental caries is still a considerable problem in many underprivileged areas, and most carious teeth are untreated, although the prevalence and severity of caries have decreased over the past few decades. ${ }^{1}$ Innovative methods are required to improve this situation. Antibiotics, metal ions, and fluorides in various vehicles have been used as alternatives to the traditional filling approach for preventing and arresting caries. Topical agents, such as silver diamine fluoride (SDF), have been concluded as effective, efficient, equitable, and safe caries-preventive agents. ${ }^{2,3}$ SDF therapy is simple and non-invasive. Its application requires no caries removal. ${ }^{4}$ The use of SDF in dentistry has gained much attention worldwide since its approval as a medical device by the US Food and Drug Administration in August 2014. Clinical trials and lab studies have supported the success of $38 \%$ SDF in arresting dental caries and in inhibiting the growth of cariogenic pathogens. ${ }^{3,5}$ 
However, SDF has been limited in its clinical application owing to its adverse effects, such as caries staining, pulpal irritation, fluorosis, and tissue irritation in the oral mucosa stemming from inadvertent contact with SDF. In dentistry, the discoloration effect on carious teeth is the most distinct deficiency of SDF, which may limit its clinical use in aesthetically demanding patients. ${ }^{6}$ Topical SDF has been suggested to form silver phosphate when applied to carious lesions, and silver phosphate readily turns black under sunlight or under the influence of reducing agents. ${ }^{7}$ Some researchers have proposed using potassium iodide after an SDF topical application to reduce the staining effect by generating silver iodide. However, silver iodide is photosensitive and turns dark with exposure to light. Ammonium hexafluorosilicate has been suggested to exclude silver and its staining effect, although it is not as effective as SDF is in managing caries. ${ }^{8}$

A possible way in which to address the displeasing aesthetic effect of SDF is to use a fluoride solution containing silver nanoparticles (AgNPs). Unlike silver ion solutions, which tend to form intense, black-colored silver chloride layers, the intrinsic optoelectronic properties of AgNPs result in significantly less tooth discoloration. AgNPs also have broad-spectrum antibacterial activities and inhibit bacterial growth at low concentrations. ${ }^{9,10}$ This is because the small sizes of the particles facilitate penetration into bacterial cells and provide maximum contact with the environment. ${ }^{11,12}$ Fluoride has been shown to enhance the remineralization of caries. Hence, fluoride solutions containing AgNPs may be used to control caries without aesthetically undesirable side effects.

A clinical trial suggested that a new anti-caries agent, nano silver fluoride (NSF), was effective in arresting dentine caries and had no staining effect on teeth. ${ }^{13}$ This NSF is shown to feature low toxicity to living cells and has a similar antibiotic property to SDF against Streptococcus mutans, the primary pathogen causing dental caries. ${ }^{14}$ However, no laboratory studies were found in the literature to investigate the remineralizing effect of fluoride with silver nanoparticles in dentine caries. Therefore, the aim of this study was to investigate the effect of a sodium fluoride (NaF) solution with our synthesized polyethylene glycol-coated silver nanoparticles (PEG-AgNPs) in remineralizing artificial dentine caries from both mineral and collagen aspects. Moreover, the optical properties of treated dentine were also assessed to identify whether fluoridated PEG-AgNPs cause any discoloration.

\section{Materials and Methods Synthesis of PEG-AgNPs}

PEG-AgNPs were successfully prepared through the onestep chemical reduction of silver acetate with the biocompatible poly (ethylene glycol) methyl ether thiol (PEG-SH). The PEG-SH was used as a reducing and coating agent for the stabilization of AgNPs. For the preparation of PEG-AgNPs, $40 \mathrm{mg}$ silver acetate and $20 \mathrm{mg}$ PEG$\mathrm{SH}$ were dissolved individually in $400 \mathrm{~mL}$ ethanol. The silver acetate solution was kept in darkness to avoid decomposition and was stirred at $4{ }^{\circ} \mathrm{C}$ until the homogeneous phase was reached. After the two solutions were mixed, the color changed immediately from colorless to light yellow, suggesting the accomplishment of the synthesis of PEG-AgNPs. The reaction mixture was stirred for $18 \mathrm{~h}$ at $4{ }^{\circ} \mathrm{C}$ under ambient light to ensure the formation and even growth of nanoparticles. PEG-AgNPs were concentrated using rotary evaporation, and a final volume of about $50 \mathrm{~mL}$ was obtained. The ethanoic solvent was exchanged to water, and PEG-AgNPs were further concentrated using an Amicon ultra centrifugal filter of MWCO $30 \mathrm{kDa}$. The unreacted starting reagents were also removed at this stage via washing three times with distilled water. To this PEG-AgNPs solution, NaF (2.5\%) was added as the fluoride source to yield afluoridated PEG-AgNP solution containing 11,310 ppm fluoride and $400 \mathrm{ppm}$ silver. Images of transmission electron microscopy showed ball-shaped silver nanoparticles with an average diameter at $2.56 \pm 0.43 \mathrm{~nm}$ in Figure 1 .

\section{Development of Artificial Dentine Caries}

This study received approval from a local institutional review board (IRB UW14-529). The protocol of our study is summarized in Figure 2. Extracted sound human third molars were collected with the patients' consent provided in hardcopy forms. The molars were stored in a $0.1 \%$ thymol solution at $4{ }^{\circ} \mathrm{C}$ and were employed for this study within one month after extraction. Eighteen dentine slices of $2 \mathrm{~mm}$ in thickness were prepared and were polished with micro-fine 4000-grit sanding paper. A stereomicroscope was used to exclude slices with cracks or other defects. Each slice was sectioned into three dentine blocks for different treatments afterward, and thus, 54 blocks were prepared. All blocks were half-covered with acid-resistant nail varnish (Clarins, Paris, France). They were then placed into a demineralization solution $(2.2 \mathrm{mM}$ $\mathrm{KH}_{2} \mathrm{PO}_{4}, 2.2 \mathrm{mM} \mathrm{CaCl}_{2}, 50 \mathrm{mM}$ acetate), $\mathrm{pH} 4.4$, at $25^{\circ} \mathrm{C}$ 

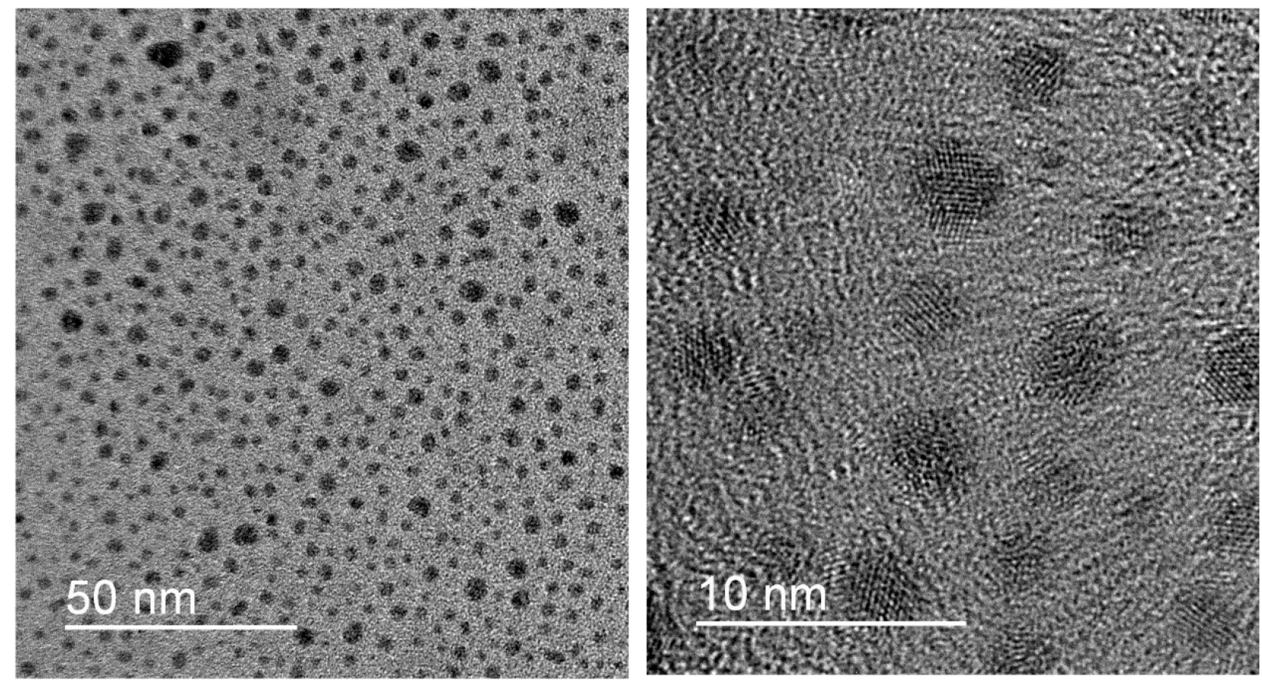

Figure I Transmission electron microscopy of silver nanoparticles.

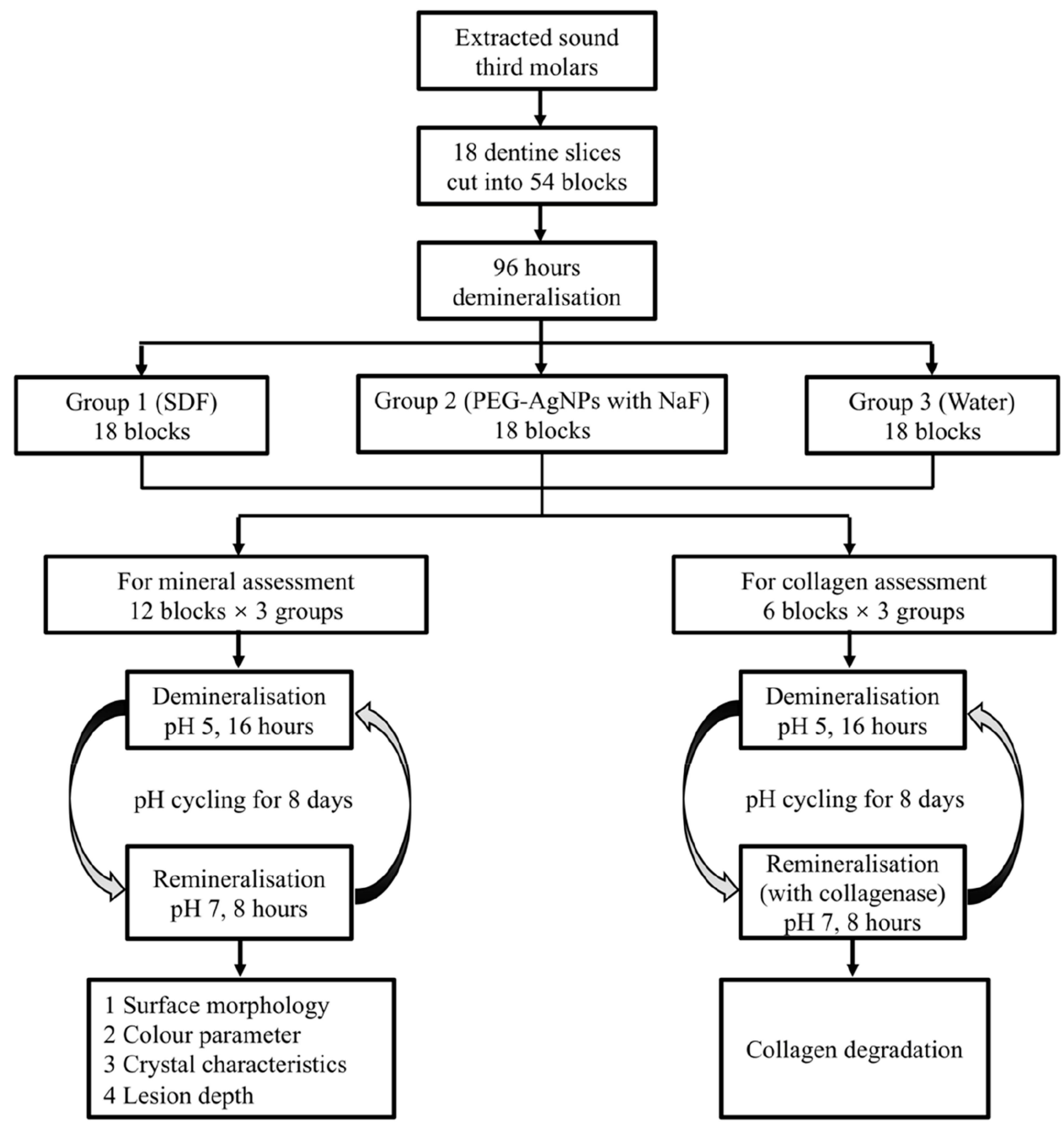

Figure 2 Flowchart of the experiment design. 
for 4 days to create artificial caries lesions that were 70-100 $\mu \mathrm{m}$ deep. ${ }^{15}$ The blocks were washed with deionized water after lesion development. Thirty-six blocks from 12 dentine slices were used for the study of dentine demineralization, and 18 blocks from six dentine slices were used to study dentine collagen degradation.

\section{Experimental Treatment}

The three dentine blocks from each slice were randomly allocated to three treatment groups. In group 1 (SDF, positive control), the blocks were dried and received topical applications of a commercial 12\% SDF solution (Cariostop 12\%; Biodinâmica Químicae Farmacêutica LTDA) containing 14,150 ppm fluoride and 80,000 ppm silver. In group 2, the blocks were treated with afluoridated PEG-AgNPs solution containing 11,310 ppm fluoride and 400 ppm silver. In group 3 , the blocks received deionized water (negative control). Micro-brushes (Micro applicator - regular, Premium Plus International Ltd., Hong Kong, China) were used to apply the solutions, and the volume of the solutions applied to each dentine surface was approximately $10 \mu \mathrm{L}$ (confirmed via a pilot test). Before the $\mathrm{pH}$ cycling procedure, the blocks were stored at $25^{\circ} \mathrm{C}$ for $30 \mathrm{~min}$.

\section{Cariogenic Challenge Using a Chemical Model}

The cariogenic challenge by means of $\mathrm{pH}$ cycling was employed to assess the demineralization effect of various treatments on dentine in the three groups. Twelve blocks of each treatment group were immersed in a demineralization solution (50 mM acetate, $1.5 \mathrm{mM} \mathrm{CaCl}_{2}, 0.9 \mathrm{mM} \mathrm{KH}_{2} \mathrm{PO}_{4}$ ) at $\mathrm{pH} 5.0$ for $16 \mathrm{~h}$, followed by $8 \mathrm{~h}$ of immersion in a remineralization solution $(150 \mathrm{mM} \mathrm{KCl}, 20 \mathrm{mM}$ 4-(2-hydroxyethyl)-1-piperazineethanesulfonic acid (HEPES), $0.9 \mathrm{mM}$ $\mathrm{KH}_{2} \mathrm{PO}_{4}, 1.5 \mathrm{mM} \mathrm{CaCl}$ ) at $\mathrm{pH}$ 7.0. The $\mathrm{pH}$ cycling procedure was performed at $25^{\circ} \mathrm{C}$ for 8 days. Solutions were freshly made before use. ${ }^{16}$

\section{Assessment of Dentine Demineralization Surface Morphology}

Four dentine blocks (two for the examination of the surface morphology and two for the examination of the crosssection topography) from each group were fixed in a $2.5 \%$ glutaraldehyde solution at $4{ }^{\circ} \mathrm{C}$ for $8 \mathrm{~h}$. They were then rinsed with deionized water ultrasonically and dehydrated with ethanol. The blocks were placed in a desiccator for critical-point drying and were sputter coated with carbon prior to observation with scanning electron microscopy (SEM; Hitachi S-4800 FEG Scanning Electron Microscope, Hitachi Ltd., Tokyo, Japan) at $5 \mathrm{kV}$ in highvacuum mode. ${ }^{17}$

\section{Colour Parameters}

The color of each dentine block was evaluated with the VITA Easyshade $^{\circledR}$ advance portable dental spectrophotometer (VITA Zahnfabrik GmbH, Bad Säckingen, Germany). Each color was elucidated three-dimensionally (3D) in space according to the Commission International del'Eclairage $\mathrm{L}^{*}$ $a^{*} b^{*}$ color system. $L^{*}$ axis represented lightness ranging from black (0) to white (100), a* axis described red $\left(+a^{*}\right)$ to green $\left(-a^{*}\right)$, and the $b^{*}$ axis represented yellow $\left(+b^{*}\right)$ to blue $\left(-b^{*}\right)$. The instrument was calibrated according to the manufacturer's instructions before assessment. The L*a*b*hue (h) and chroma $(\mathrm{C})$ values were measured in triplicate, and the average values were recorded. The difference in the color $(\Delta \mathrm{E})$ between experimental groups (groups 1 and 2) and the negative control group (group 3 ) was calculated based on the mathematical equation of $\Delta \mathrm{E}^{*}=\left[(\Delta \mathrm{L})^{2}+(\Delta \mathrm{a})^{2}+(\Delta \mathrm{b})^{2}\right]^{1 / 2}$. The perceptibility threshold of $\Delta \mathrm{E}$, where the tooth color change was clinically visible to the naked eye, was set at 3.7 units. $^{8}$

\section{Crystal Characteristics}

Two dentine blocks from each group were used for x-ray diffraction (XRD) analysis via a diffractometer Bruker D8 Advance $x$-ray powder with $\mathrm{CuKa}(1=1.5418 \AA)$ radiation at $40 \mathrm{kV}$ and $40 \mathrm{~mA}$. The data were collected with the following parameters: step size $=0.05^{\circ}, 2 \mathrm{q}$ range $=$ $20-60^{\circ}$, and scan speed $=30$ seconds/step. A matching search database of the International Centre for Diffraction Data (ICDD, PDF-2 Release 2004) was employed to check the indexing and purity of the chemical phase. The diffraction patterns were studied using the Bruker DIFFRACplus EVA program. ${ }^{18}$

\section{Lesion Depth}

According to our previous studies, the mean lesion depth of the test group was expected to be $150 \mu \mathrm{m}$. The detected difference was assumed to be at least $100 \mu \mathrm{m}$. The common standard deviation was assumed to be $60 \mu \mathrm{m}$ with power at 0.80 and $\alpha$ at 0.05 , and the sample size was six in each group. Dentine blocks ( $n=6$ per group) were examined to assess lesion depth using a SkyScan 1076 microCT (SkyScan, Antwerp, Belgium) at $100 \mathrm{kV}$ and $80 \mu \mathrm{A}$. The blocks were scanned with the highest spatial resolution of $9 \mu \mathrm{m}$. NRecon reconstruction software (SkyScan, 
Antwerp, Belgium) was used to reconstruct the scanning results of each block. After reconstruction, images of the blocks were viewed using CTAn (SkyScan, Antwerp, Belgium). Cross-sectional images displaying the lesion area of each block were located from the reconstructed 3-D images. Ten images were selected from these lesion images. The lesion depth was determined using image analysis software (Image $\mathrm{J}$; National Institutes of Health, USA).

\section{Collagen Degradation}

The dentine blocks ( $\mathrm{n}=6$ per group) were $\mathrm{pH}$ cycled at $37^{\circ} \mathrm{C}$ for 8 days via immersion individually in a demineralization solution (50 mM acetate, $1.5 \mathrm{mM} \mathrm{CaCl}_{2}, 0.9 \mathrm{mM} \mathrm{KH}_{2} \mathrm{PO}_{4}$ ) at $\mathrm{pH} 5.0$ for $16 \mathrm{~h}$ and in a remineralization solution $(150 \mathrm{mM}$ $\mathrm{KCl}, 20 \mathrm{mM}$ HEPES, $0.9 \mathrm{mM} \mathrm{K \textrm {K } _ { 2 }} \mathrm{PO}_{4}, 7.5 \mathrm{U} / \mathrm{mL}$ highly purified collagenase type VII from Clostridium histolyticum (C-0773, Sigma Chemical Co., St. Louis, MO, USA), $1.5 \mathrm{mM}$ $\mathrm{CaCl}_{2}$ ) at $\mathrm{pH} 7.0$ for $8 \mathrm{~h}$. All solutions were freshly prepared before use. The amount of amino acid hydroxyproline was estimated to determine the collagen degradation. After $\mathrm{pH}$ cycling, the remineralization solution of each block was collected. The total remineralization solution was concentrated and solidified at a high drying rate using a Savant SpeedVac Concentrator (Thermo Scientific, Waltham, UK). The concentrated samples were then dissolved in $100 \mu \mathrm{L}$ distilled water and hydrolyzed via autoclaving at $120^{\circ} \mathrm{C}$ for $20 \mathrm{~min}$. The hydrolyzed samples were gently mixed with $0.056 \mathrm{M}$ chloramine-T reagent and were used for oxidation at $25{ }^{\circ} \mathrm{C}$ for $25 \mathrm{~min}$. After $1 \mathrm{M}$ Ehrlich's aldehyde reagent was added to each sample and incubated at $65^{\circ} \mathrm{C}$ for $20 \mathrm{~min}$, the chromophore was generated. A hydroxyproline standard solution containing 2-20 $\mu \mathrm{g}$ HYP was also created. ${ }^{15}$ The absorbance of each sample was measured at $550 \mathrm{~nm}$ using a spectrophotometer (SpectraMax 340, Sunnyvale, CA, USA). The coefficient of determination for the standard curve of the known hydroxyproline solution was 0.95 .

\section{Statistical Analysis}

The characteristics of crystal by XRD and surface morphology by SEM were observed and not subjected to statistical analyses. The normality of all data were checked using the Shapiro-Wilk test. One-way analysis of variance (ANOVA) and the Bonferroni post hoc test were employed to analyze the change of the color parameters, the lesion depth of the dentine, and the concentration of hydroxyproline in remineralization solutions among the three treatment groups. The level of significance was set to 0.05. Analyses were performed using SPSS Statistics - V20.0 computer software (IBM Corporation, Armonk, NY, USA).

\section{Results}

An observation under SEM revealed that the dentine surface of SDF-treated dentine and NaF with PEGAgNPs-treated dentine was relatively intact and dense (Figure 3). In group 3, the exposure of dentine collagen fibers was considerably apparent, and collagen fibers were distributed intricately (Figure 3). The features of collagen fibers were also verified through the cross-sectional images (Figure 4). Cross-sectional images in the groups of SDF and fluoridated PEG-AgNPs exhibited the formation of compact granuliform structures of ball-shaped particles in the inter-tubular region, which are not observed in group 3 .

The color parameters of the dentine blocks in the three groups are shown in Table 1. No significant differences were found in color parameters $\mathrm{L}^{*} \mathrm{C}$, and $\mathrm{h}$ between group 2 (PEG$\mathrm{AgNPs}+\mathrm{NaF})$ and group 3 (Water) $(p>0.05)$. On the contrary, the $\mathrm{L}^{*} \mathrm{C}$, and $\mathrm{h}$ values of dentine blocks in group 1 (SDF) were significantly lower than those of the two other groups $(p<0.001)$. The blocks treated with SDF displayed a perceptible color difference of $\Delta \mathrm{E}^{*}=85.0$, whereas the blocks treated with fluoridated PEG-AgNPs did not show a noticeable color variation with $\Delta \mathrm{E}^{*}=2.2$. The data indicated that fluoridated PEG-AgNPs did not introduce any adverse color effect on dentine, but SDF caused distinct discoloration.

The crystal characteristics on the dentine surfaces were examined using XRD analysis. The typical XRD spectra (Figure 5) of the dentine blocks in the three groups showed the presence of hydroxyapatite (HAP) crystallized at $31.8^{\circ}$ (211), $32.9^{\circ}(300)$, and $39.8^{\circ}$ (310). However, the main diffraction peaks in group 3 (Water) were wider and lower compared with the other two groups. Apart from hydroxyapatite, fluroapatite (FAP) was detected at $25.8^{\circ}(002), 31.9^{\circ}$ (211), $32.3^{\circ}(112)$, and $33.1^{\circ}$ (300) in groups 1 (SDF) and 2 (PEG-AgNPs). In addition, the characteristic peaks at $27.8^{\circ}$, $32.2^{\circ}$, and $46.2^{\circ}$ were coincident with Bragg reflections of silver chloride, suggesting the formation of silver chloride in group 1 (SDF). In addition, the representative peaks in group 1 (SDF) at $38.1^{\circ}(111)$ and $44.3^{\circ}(200)$ indicated that metallic silver was formed on the dentine surfaces.

Representative images of the micro-computed tomography of the three groups are displayed in Figure 6. The mean lesion depth $( \pm \mathrm{SD})$ of artificial caries in group 1 (SDF) and group 2 (PEG-AgNPs $+\mathrm{NaF}$ ) were $118 \pm 7 \mu \mathrm{m}$ and $121 \pm 14$, respectively, which were significantly lower 

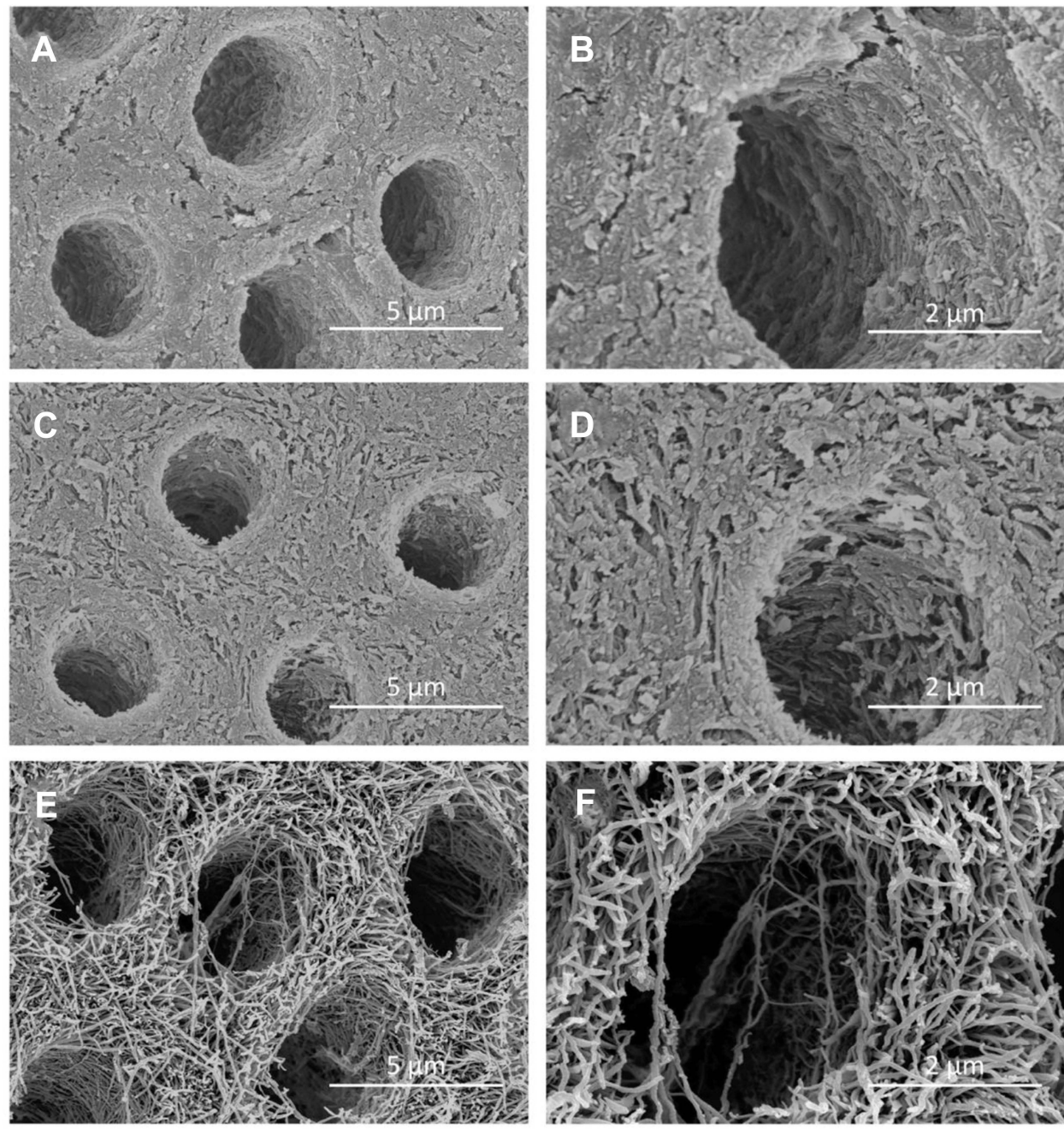

Figure 3 SEM images of surface morphology of artificial dentine caries.

Notes: (A) SDF (8,000x); (B) SDF (20,000x); (C) PEG-AgNPs with NaF (8,000x); (D) PEG-AgNPs with NaF (20,000x); (E) Water (8,000x); (F) Water (20,000×).

than that of group 3 (Water) $(p<0.001)$ (Table 2). The amount of degraded collagen is directly in proportion to the concentration of hydroxyproline of each dentine block. The concentration of hydroxyproline was significantly higher in group 3 (Water, $335 \pm 21$ ) than in group 2 (SDF, $122 \pm 28$ ) and group 3 (PEG-AgNPs $+\mathrm{NaF}, 146 \pm$ 22) $(p<0.001)$.

\section{Discussion}

A review concluded that the topical application of SDF is a cost-effective, user-friendly, and non-invasive approach in caries management. ${ }^{19}$ Clinical trials demonstrated that SDF is effective in preventing and arresting dental caries in primary teeth and permanent teeth. ${ }^{5}$ Laboratory studies showed that SDF increases the resistance of dentine to demineralization and collagen degradation. ${ }^{20}$ However,
SDF is limited in its clinical use due to staining carious tissue black. A clinical trial that suggested that NSF could arrest active dentine caries and not cause tooth discoloration. ${ }^{13}$ However, the mode of action of NSF in arresting caries has not yet been clarified. The concentrations of silver and fluoride used in Dos Santos Jr.'s study were approximately $400 \mathrm{ppm}$ and 2,275 ppm, respectively. ${ }^{21}$ However, our pilot study found that 2,275 ppm fluoride was insufficient for remineralizing the demineralized dentine. The fluoride concentration at around $10,000 \mathrm{ppm}$ or above displayed favorable remineralizing effects. $^{22}$ Thus, silver nanoparticles were selected at a concentration of $400 \mathrm{ppm}$ and $2.5 \%$ sodium fluoride (11,310 ppm fluoride) in our study. A commercially available $12 \%$ SDF with a similar fluoride concentration $(14,150$ ppm fluoride) was used as a positive control in this study. 

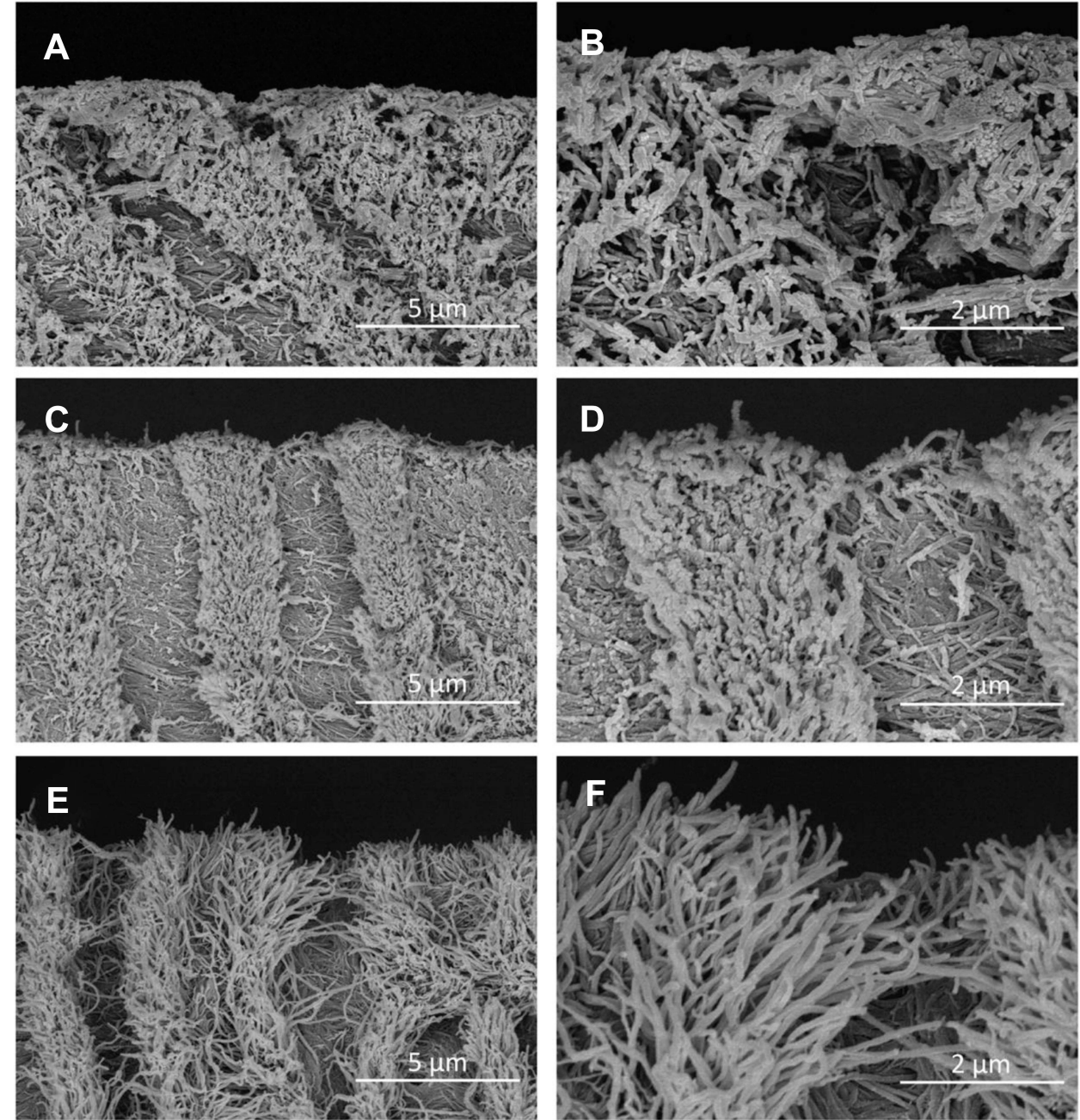

Figure 4 SEM images of cross-section of artificial dentine caries.

Notes: (A) SDF (8,000x); (B) SDF (20,000x); (C) PEG-AgNPs with NaF (8,000x); (D) PEG-AgNPs with NaF (20,000x); (E) Water (8,000×); (F) Water (20,000x).

Three conditions are essential for remineralizing demineralized dentin: mineral crystals should serve as growth centers; calcium and phosphorus should be supplied as mineral sources; and a collagen structure acting as a scaffold should be intact for mineral crystals to grow on. ${ }^{6}$ The cross-sectional images of SEM reviewed that compact granuliform structures of ball-shaped grains were formed in the inter-tubular area in the groups of

Table I Mean (SD) of Colour Parameters of Dentine After Treatments

\begin{tabular}{|l|l|l|l|l|l|}
\hline \multirow{2}{*}{} & \multicolumn{2}{|l|}{ Group } & \multirow{2}{*}{$\boldsymbol{p}$ value } & \multirow{2}{*}{ Bonferroni } \\
\cline { 2 - 4 } & I (SDF) & 2 (PEG-AgNPs+NaF) & $\mathbf{3}$ (Water) & & \\
\hline $\mathrm{L}^{*}$ & $\mathrm{I} 6.1(0.7)$ & $92.4(8.0)$ & $93.1(8.0)$ & $<0.00 \mathrm{I}$ & $\mathrm{I}<2,3$ \\
$\mathrm{C}$ & $7.6(0.5)$ & $40.9(6.2)$ & $42.9(6.5)$ & $<0.00 \mathrm{I}$ & $\mathrm{I}<2,3$ \\
$\mathrm{~h}$ & $66.7(1.3)$ & $90.1(2.5)$ & $90.2(1.9)$ & $<0.00 \mathrm{I}$ & $\mathrm{I}<2,3$ \\
$\mathrm{a}^{*}$ & $3.0(0.2)$ & $-0.0(1.6)$ & $-0.2(1.4)$ & N/A & N/A \\
$\mathrm{b}^{*}$ & $7.0(0.5)$ & $40.9(6.2)$ & $42.9(6.5)$ & N/A & N/A \\
$\Delta \mathrm{E}^{*}$ & 85.0 & 2.2 & N/A & N/A & N/A \\
\hline
\end{tabular}

Notes: $L^{*}$ axis represented lightness ranged from black $(0)$ to white $(100)$, $a^{*}$ axis described red $\left(+a^{*}\right)$ to green $\left(-a^{*}\right)$, b* axis represented yellow $\left(+b^{*}\right)$ to blue (- $\left.\mathrm{b}^{*}\right) \mathrm{h}$ means hue, and $\mathrm{C}$ means chroma, $\Delta \mathrm{E}^{*}$ was the difference of colour calculated based on the equation $\Delta \mathrm{E}^{*}=\left[(\Delta \mathrm{L})^{2}+(\Delta \mathrm{a})^{2}+(\Delta \mathrm{b})^{2}\right]^{1 / 2}$. Abbreviations: SDF, silver diamine fluoride; PEG-AgNPs polyethylene glycol-coated silver nanoparticles, N/A, not applicable. 


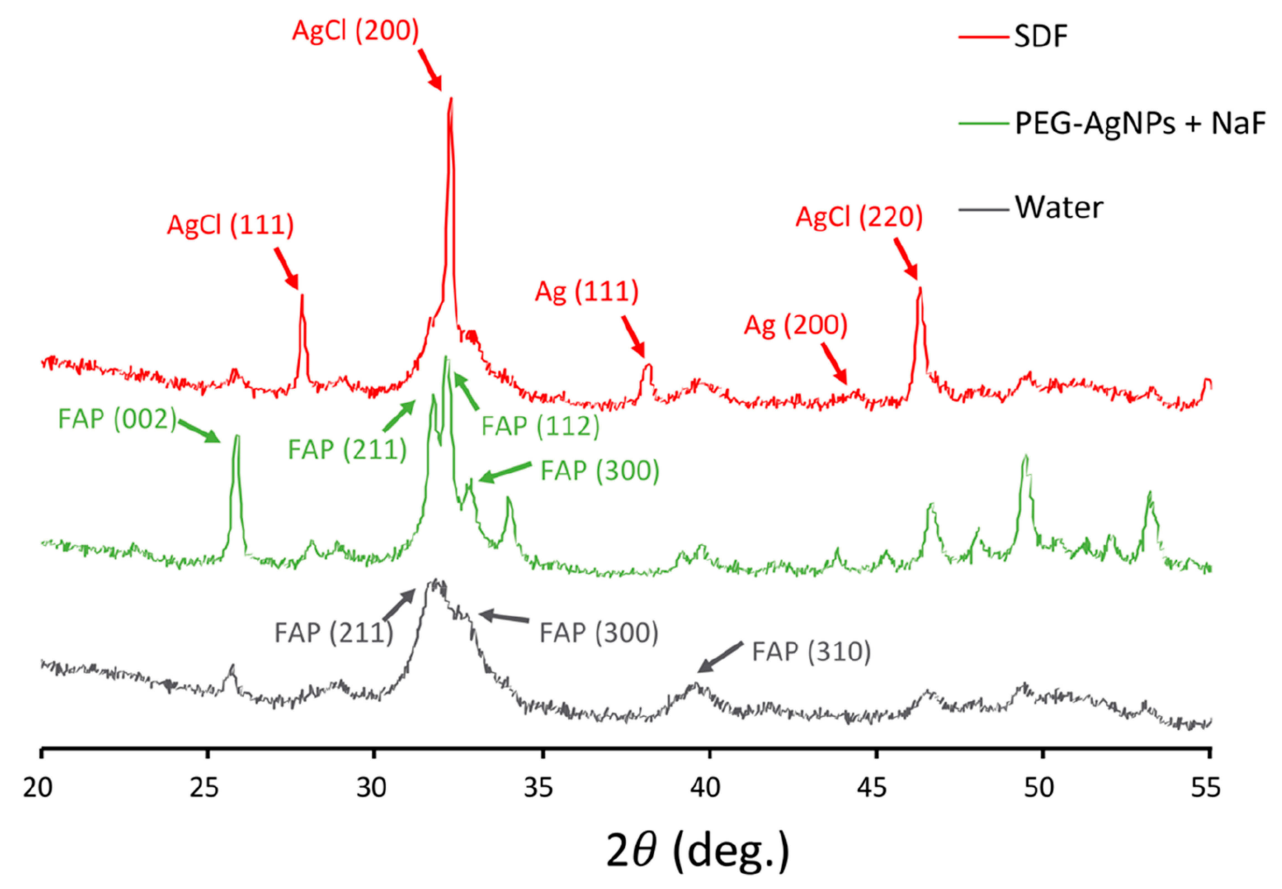

Figure 5 Typical X-ray diffraction patterns of the three groups.

SDF and fluoridated PEG-AgNPs. This suggested that an extra-fibrillar mineral was formed, and the remineralization of demineralized dentine was facilitated in the groups of SDF and fluoridated PEG-AgNPs. An observation from SEM was consistent with the assessment of dentine collagen degradation in the present study. Our findings showed that the collagen degradation of dentine was partially inhibited by SDF or NaF with PEG-AgNPs. Dentine type I collagen accounts for approximately $90 \%$ of organic component in dentine, and the residual part is composed of noncollagenous proteins. ${ }^{19}$ Thus, the process of dentine caries cannot easily be explained by mineral loss due to bacterial acid corrosion. The organic matrix of dentine should play an important role in the progression of dentine caries. It is plausible that PEGAgNPs could interact with exposed dentine collagen so as to inhibit collagenase activity.

The aesthetic appearance of dental treatment is the main concern for patients. Tooth discoloration stemming from the topical application of SDF is a significant disadvantage, whereas the staining effect of dental tissues resulting from fluoridated PEG-AgNPs has not been quantified. In this study, instrumental measurements were used to quantify color characteristics precisely with high reproducibility; this was done rather than subjectively assessing color differences by the naked eye. The range of the clinical perceptibility threshold was from $\Delta \mathrm{E}^{*}=1$ to 3.7 , whereas color differences that were considered to be accepted clinically ranged from $\Delta \mathrm{E}^{*}=2.7$ to $6.8 .^{23}$ Based on this, color differences resulting from fluoridated PEG-AgNPs $\left(\Delta \mathrm{E}^{*}=2.2\right)$ cannot be detected visually. The discoloration of dentine stemming from $\operatorname{SDF}\left(\Delta \mathrm{E}^{*}=85.0\right)$ would not be accepted clinically, as it was approximately 10 times higher than the upper acceptability threshold was.

The first reaction between SDF and hydroxyapatite is the formation of silver phosphate and calcium fluoride. After the subsequent separation of calcium fluoride and calcium, fluroapatite can be formed gradually. Fluroapatite is produced when the fluoride concentration is low. In addition, calcium fluoride is produced with a high concentration of fluoride. Fluroapatite is less acid soluble than hydroxyapatite. ${ }^{24}$ Because the time for demineralization $(\mathrm{pH}$ at 5) is much longer than that for remineralization ( $\mathrm{pH}$ at 7) of $\mathrm{pH}$ cycling, the formation of fluroapatite has a higher chance of occurring. This could be the reason why fluroapatite was detected in groups SDF and fluoridated PEG-AgNPs by XRD. Calcium fluoride, which is less acid resistant than fluroapatite is, is considered to be a pH-regulated slow-release fluoride reservoir at a time of reactions. The formation of fluroapatite can be facilitated when a hydrogen phosphate ion is absorbed onto the calcium fluoride crystal surface. ${ }^{6}$ In our study, calcium fluoride was not detected using XRD. Calcium fluoride can disintegrate and disappear easily after washing by water. Additionally, when heavy atoms, such as silver atoms, exist, 

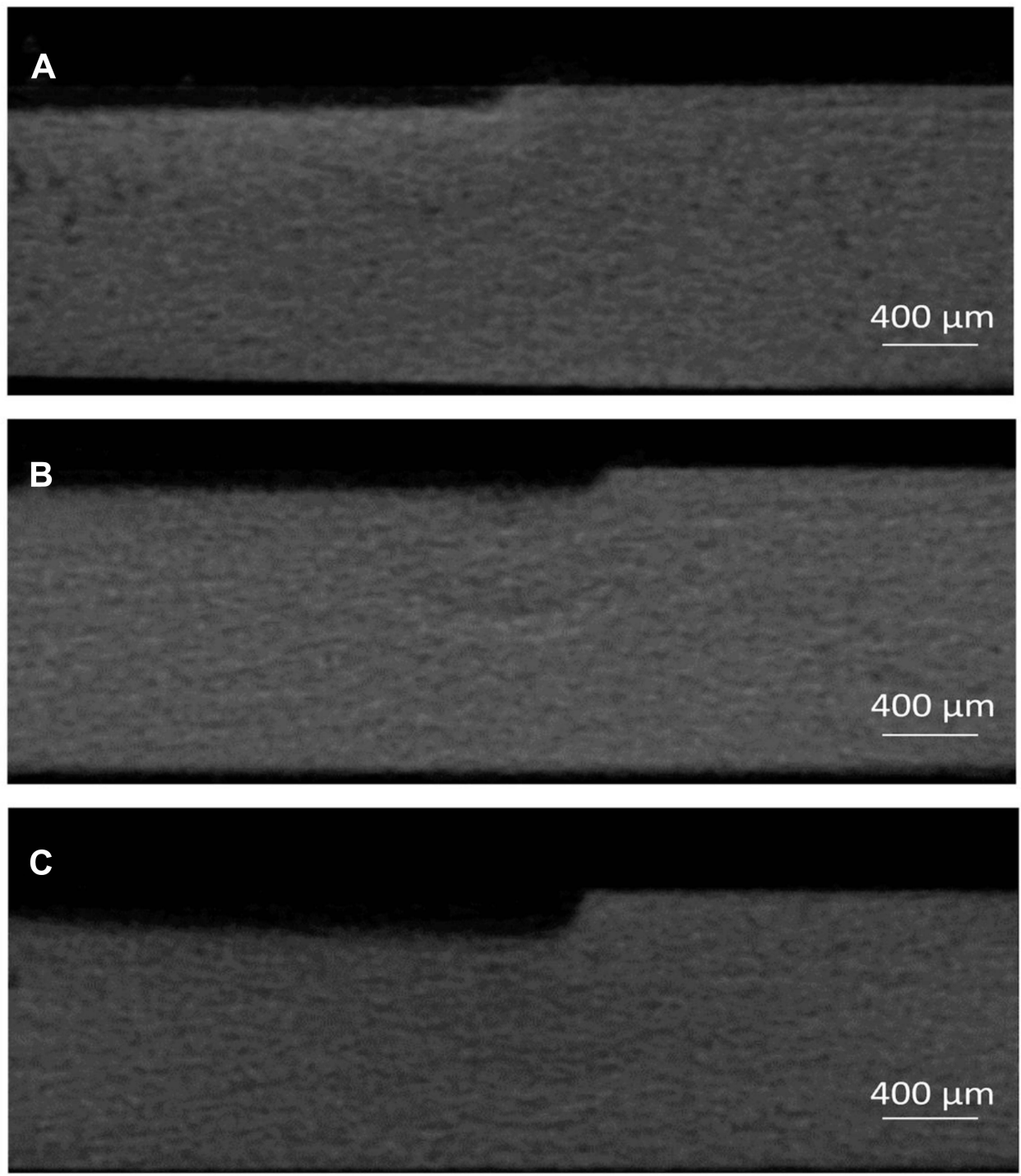

Figure 6 Typical micro-CT images of cross-section of artificial dentine caries.

Notes: Left side: lesion body; right side: internal control. (A) SDF; (B) PEG-AgNPs with NaF; (C) Water.

the signal of calcium fluoride may weaken. In group 1 (SDF), metallic silver was formed on SDF-treated dentine, but it could not be detected in dentine receiving fluoridated PEGAgNPs. The reason for this may be that the application of fluoridated PEG-AgNPs did not cause staining on dentine surfaces. Because the solubility of silver phosphate $\left(\mathrm{Ag}_{3}\right.$
$\mathrm{PO}_{4}, 6.4 \times 10^{-3} \mathrm{~g} / 100 \mathrm{~mL}$ ) is higher than that of silver chloride $\left(\mathrm{AgCl}, 1.9 \times 10^{-4} \mathrm{~g} / 100 \mathrm{~mL}\right)$, silver phosphate could react to the alkali chlorides in remineralization solutions to form silver chloride. This could explain why silver chloride was detected as the principal precipitate in the SDF group using XRD in this study.

Table 2 Mean (SD) Lesion Depth and Hydroxyproline Concentration of the Three Groups

\begin{tabular}{|l|l|l|l|l|l|}
\hline \multirow{2}{*}{ Assessment of Demineralisation } & \multicolumn{2}{|l|}{ Group } & \multirow{2}{*}{ P value } & \multirow{2}{*}{ Bonferroni } \\
\cline { 2 - 5 } & I (SDF) & 2 (PEG-AgNPs + NaF) & $\mathbf{3}$ (Water) & & \\
\hline Lesion depth $(\mu \mathrm{m})$ & $\mathrm{II}(7)$ & I2I (14) & $339(20)$ & $<0.00 \mathrm{I}$ & $\mathrm{I}, 2<3$ \\
Hydroxyproline concentration & $\mathrm{I} 22(28)$ & I $46(22)$ & $335(2 \mathrm{I})$ & $<0.00 \mathrm{I}$ & $\mathrm{I}, 2<3$ \\
\hline
\end{tabular}

Abbreviations: SDF, silver diamine fluoride; PEG-AgNPs, fluoridated polyethylene glycol-coated silver nanoparticles. 
Silver nanoparticles had strong antibacterial and antibiofilm properties, providing long-lasting protection against bacteria. ${ }^{25,26}$ Vieira Costa and Silva et al evaluated the antibacterial and cytotoxic activity of NSF against Streptococcus mutans, and they found that NSF was a bacteriostatic and bactericidal compound with a similar antimicrobial property to SDF and low toxicity to living cells. ${ }^{27}$ Further research can be conducted to evaluate the effect of fluoridated PEG-AgNPs on multi-species cariogenic biofilm.

\section{Conclusion}

The 2.5\% NaF with PEG-AgNPs and 12\% SDF had a similar remineralizing effect in remineralizing artificial dentine caries and in inhibiting collagen degradation. Because $2.5 \% \mathrm{NaF}$ with PEG-AgNPs does not stain dentine caries, it may be a better anti-caries agent for addressing the disadvantage of SDF.

\section{Ethics and Consent Statement}

This study received approval from the Institutional Review Board at the University of Hong Kong under process number IRB UW14-529. Extracted sound human third molars were collected with the patients' consent provided in hardcopy forms.

\section{Acknowledgment}

This study was supported by the National Natural Science Foundation of China (NSFC) - General Program (2018) 81870812 and the General Research Fund (GRF) 17100218 of the Research Grant Council, Hong Kong.

\section{Disclosure}

The authors declare that they have no conflicts of interest in this work.

\section{References}

1. Duangthip D, Chu CH, Lo ECM. A randomized clinical trial on arresting dentine caries in preschool children by topical fluorides18 month results. J Dent. 2016;44:57-63. doi:10.1016/j.jdent. 2015.05.006

2. Duangthip D, Gao SS, Chen KJ, Lo ECM, Chu CH. Oral health-related quality of life of preschool children receiving silver diamine fluoride therapy: a prospective 6-month study. J Dent. 2019;81:27-32. doi:10.1016/j.jdent.2018.12.004

3. Yu OY, Mei ML, Zhao IS, Li QL, Lo EC, Chu CH. Remineralisation of enamel with silver diamine fluoride and sodium fluoride. Dent Mater. 2018;34(12):e344-e352.

4. Gao SS, Zhao IS, Hiraishi N, et al. Clinical trials of silver diamine fluoride in arresting caries among children: a systematic review. JDR Clin Transl Res. 2016;1(3):201-210. doi:10.1177/2380084416661474
5. Gao SS, Chen KJ, Duangthip D, Wong MCM, Lo ECM, Chu CH. Preventing early childhood caries with silver diamine fluoride: study protocol for a randomised clinical trial. Trials. 2020;21(1):140. doi:10.1186/s13063-020-4088-7

6. Mei ML, Lo ECM, Chu CH. Arresting dentine caries with silver diamine fluoride: what's behind it? J Dent Res. 2018;97(7):751-758. doi: $10.1177 / 0022034518774783$

7. Yu OY, Zhao IS, Mei ML, Lo ECM, Chu CH. Caries-arresting effects of silver diamine fluoride and sodium fluoride on dentine caries lesions. J Dent. 2018;78:65-71. doi:10.1016/j.jdent.2018.08.007

8. Zhao IS, Mei ML, Burrow MF, Lo EC, Chu CH. Effect of silver diamine fluoride and potassium iodide treatment on secondary caries prevention and tooth discolouration in cervical glass ionomer cement restoration. Int J Mol Sci. 2017;18(2). doi:10.3390/ijms18020340

9. Pugazhendhi A, Prabakar D, Jacob JM, Karuppusamy I, Saratale RG. Synthesis and characterization of silver nanoparticles using Gelidium amansii and its antimicrobial property against various pathogenic bacteria. Microb Pathog. 2018;114:41-45. doi:10.1016/j.micpath. 2017.11.013

10. Saravanan M, Barik SK, Mubarakali D, Prakash P, Pugazhendhi A. Synthesis of silver nanoparticles from Bacillus brevis (NCIM 2533) and their antibacterial activity against pathogenic bacteria. Microb Pathog. 2018;116:221-226. doi:10.1016/j.micpath.2018.01.038

11. Oves M, Aslam M, Rauf MA, et al. Antimicrobial and anticancer activities of silver nanoparticles synthesized from the root hair extract of Phoenix dactylifera. Mater Sci Eng C. 2018;89:429-443. doi:10.1016/j.msec.2018.03.035

12. Shanmuganathan R, Karuppusamy I, Saravanan M, et al. Synthesis of silver nanoparticles and their biomedical applications a comprehensive review. Curr Pharm Des. 2019;25(24):2650-2660. doi:10.2174/1381612825666190708185506

13. Tirupathi S, Svsg N, Rajasekhar S, Nuvvula S. Comparative cariostatic efficacy of a novel Nano-silver fluoride varnish with $38 \%$ silver diamine fluoride varnish a double-blind randomized clinical trial. $J$ clin exp dent. 2019;11(2):e105-e112. doi:10.4317/jced.54995

14. Teixeira JA, Silva A, Dos Santos Junior VE, et al. Effects of a new nano-silver fluoride-containing dentifrice on demineralization of enamel and streptococcus mutans adhesion and acidogenicity. Int J Dent. 2018;2018:1351925. doi:10.1155/2018/1351925

15. Zhao IS, Mei ML, Li QL, Lo ECM, Chu CH. Arresting simulated dentine caries with adjunctive application of silver nitrate solution and sodium fluoride varnish: an in vitro study. Int Dent J. 2017;67 (4):206-214. doi:10.1111/idj.12291

16. Yu OY, Mei ML, Zhao IS, Lo EC, Chu CH. Effects of fluoride on two chemical models of enamel demineralization. Mater. 2017;10:11.

17. Yin IX, Yu OY, Zhao IS, et al. Developing biocompatible silver nanoparticles using epigallocatechin gallate for dental use. Arch Oral Biol. 2019;102:106-112. doi:10.1016/j.archoralbio.2019.03.022

18. Yu OY, Mei ML, Zhao IS, Li Q-L, Lo EC-M, Chu C-H. Remineralisation of enamel with silver diamine fluoride and sodium fluoride. Dent Mater. 2018;34(12):e344-e352. doi:10.1016/j.dental.2018.10.007

19. Zhao IS, Gao SS, Hiraishi N, et al. Mechanisms of silver diamine fluoride on arresting caries: a literature review. Int Dent J. 2018;68 (2):67-76. doi:10.1111/idj.12320

20. Zhao IS, Mei ML, Burrow MF, Lo EC, Chu CH. Prevention of secondary caries using silver diamine fluoride treatment and casein phosphopeptide-amorphous calcium phosphate modified glass-ionomer cement. $J$ Dent. 2017;57:38-44. doi:10.1016/j. jdent.2016.12.001

21. VEd S Jr, Vasconcelos Filho A, Targino AGR, et al. A new "silverbullet" to treat caries in children-nano silver fluoride: a randomised clinical trial. J Dent. 2014;42(8):945-951. doi:10.1016/j.jdent. 2014.05.017

22. Duangthip D, Chen K, Gao SS, Lo E, Chu C. Managing early childhood caries with atraumatic restorative treatment and topical silver and fluoride agents. Int J Environ Res Public Health. 2017;14(10). 
23. Westland S, Luo W, Li Y, Pan Q, Joiner A. Investigation of the perceptual thresholds of tooth whiteness. J Dent. 2017;67s:S11-s14. doi:10.1016/j.jdent.2017.09.013

24. Yu OY, Zhao IS, Mei ML, Lo EC, Chu CH. Effect of silver nitrate and sodium fluoride with tri-calcium phosphate on streptococcus mutans and demineralised dentine. Int J Mol Sci. 2018;19(5):1288. doi:10.3390/ijms 19051288

25. Saravanan M, Arokiyaraj S, Lakshmi T, Pugazhendhi A. Synthesis of silver nanoparticles from Phenerochaete chrysosporium (MTCC-787) and their antibacterial activity against human pathogenic bacteria. Microb Pathog. 2018;117:68-72. doi:10.1016/j.micpath.2018.02.008
26. Shanmuganathan R, MubarakAli D, Prabakar D, et al. An enhancement of antimicrobial efficacy of biogenic and ceftriaxone-conjugated silver nanoparticles: green approach. Environ Sci Pollut Res Int. 2018;25 (11):10362-10370. doi:10.1007/s11356-017-9367-9

27. Vieira Costa e Silva A, Teixeira JA, Mota CCBO, et al. In vitro morphological, optical and microbiological evaluation of nanosilver fluoride in the remineralization of deciduous teeth enamel. Nanotechnol Rev. 2018;7(6):509-520. doi:10.1515/ntrev-2018-0083

\section{Publish your work in this journal}

The International Journal of Nanomedicine is an international, peerreviewed journal focusing on the application of nanotechnology in diagnostics, therapeutics, and drug delivery systems throughout the biomedical field. This journal is indexed on PubMed Central, MedLine, CAS, SciSearch ${ }^{\mathbb{R}}$, Current Contents ${ }^{\mathbb{R}} /$ Clinical Medicine,
Journal Citation Reports/Science Edition, EMBase, Scopus and the Elsevier Bibliographic databases. The manuscript management system is completely online and includes a very quick and fair peer-review system, which is all easy to use. Visit http://www.dovepress.com/ testimonials.php to read real quotes from published authors. 